\title{
METODOLOGÍAS APLICABLES A LA DETERMINACIÓN DE VELOCIDAD Y DIRECCIÓN DEL FLUJO DE AGUAS SUBTERRÁNEAS MEDIANTE TRAZADORES ARTIFICIALES
}

\author{
Methodologies applied to determination of groundwater velocities and \\ flows direction through the aid of artificial tracers
}

\author{
Carlos Sebastián Calvo ${ }^{1}$
}

PRESENTACIÓN: OCTUBRE 2016

ACEPTACIÓN: NOVIEMBRE 2016

\begin{abstract}
The present report, in the frame of the field studies of the Project "Evaluation of the Groundwater Resources of Peru", methodologies and techniques developed for onsite artificial tracer aided measurements of groundwater flow velocities and flow directions, are presented here. Horizontal flows are computed through labeling of the whole water column which is coated with a holed pipe in its entire length, below the piezometric level. On the other hand, flow directions are found by the aid of a particular radiotracer with adsorption properties in electroplated metals, by monitoring its concentration in terms of radiation count rate at the inside perimeter of the well. Concentration monitoring inside the well, requires the installation of a proper metal net positioned and fixed with a well-known orientation inside the well, prior to the experiment.
\end{abstract}

Keywords: Unique well technique, artificial tracers, groundwater flow

\begin{abstract}
RESUMEN
En el presente trabajo, que forma parte de la ejecución del proyecto «Evaluación del Potencial de Recursos Hídricos Subterráneos del Perú», se describen los aspectos metodológicos de las técnicas desarrolladas para las mediciones de velocidades y direcciones de flujo de agua subterráneas mediante el uso de trazadores artificiales. Los flujos horizontales se determinan a través del marcado de toda la columna de agua que proviene de un pozo revestido con tubería ranurada en toda su longitud por debajo del nivel piezométrico. Al mismo tiempo, las direcciones de flujo determinan, mediante el uso de un trazador que tenga propiedades de adsorción en metales, y monitorean su concentración en el perímetro interior del pozo. Este último se refiere a la concentración del trazador al interior del pozo y requiere de una malla metálica cilíndrica que debe ser colocada previamente adentro y fijada con una orientación determinada.
\end{abstract}

Palabras clave: Técnica de pozo único, trazadores artificiales, flujo de agua subterránea.

1 Facultad de Ingeniería, Universidad Ricardo Palma Lima, Perú. E-mail: csebastiancalvo@gmail.com 


\section{INTRODUCCIÓN}

Se presenta la metodología aplicable para la determinación de velocidades y direcciones de flujo de aguas subterráneas en pozo único, las cuales utilizan técnicas de trazadores convencionales y radiactivos.

Para que estas puedan aplicarse, se precisa disponer de pozos, a través de los cuales el agua pueda circular más o menos libremente, es decir, pozos no entubados, si la litología del terreno lo permite, o pozos revestidos con tubería ranurada a lo largo del tramo abarcado por la zona saturada (lo más recomendable es realizarlo en todo el tramo). El número de ranuras debe ser tal que el porcentaje de superficie bajo ese efecto se encuentre comprendido entre 0,5 y $3 \%$, con el objeto de que la permeabilidad del tubo de revestimiento sea elevada.

El diámetro interno ideal de los pozos es del orden de 3 a 4 pulgadas, incluso cuando los ensayos que se describen más adelante pueden realizarse también en pozos de 2 pulgadas de diámetro interno. Por razones obvias, los piezómetros provistos de tubería ranurada solo en los 2 o 3 últimos metros no son apropiados, si bien, en algunos casos, pueden proporcionar alguna información. Como es lógico, el flujo de agua existente en el interior de un pozo puede ser horizontal o vertical.

\section{OBJETIVO}

Determinar las velocidades y direcciones de flujo en un sector del valle de Lanchas, utilizando técnicas de trazadores aplicadas a pozo único, a fin de contribuir en la solución del problema de gestión del acuífero.

\section{PROBLEMÁTICA}

Los agricultores de la parte baja del valle de Lanchas se sienten afectados por las empresas que sobreexplotan el recurso hídrico del acuífero. Con la hidrología tradicional, no se ha conseguido, hasta el momento, determinar las direcciones de flujo en el sector del valle de Lanchas a fin de probar o descartar las afirmaciones de los labriegos. La inexistencia de pozos múltiples impide elaborar un mapa de isohipsas, el cual daría información sobre las direcciones de flujo de las aguas subterráneas.

Al determinar los parámetros de transporte del agua subterránea, como las velocidades y direcciones de flujo, la Autoridad Nacional del Agua (ANA) puede resolver el problema de gestión del acuífero atendiendo a las frecuentes quejas de los agricultores de la parte baja y coadyuvando a la solución de los conflictos entre estos y dicha institución.

Las figuras la y 1 b muestran el ámbito de estudios del presente trabajo y el área específica de las evaluaciones, materia del presente reporte.

\section{FUNDAMENTOS TEÓRICOS Y TÉCNICAS EXPERIMENTALES}

\subsection{Para la medición de los flujos horizontales (velocidad de flujo de aguas subterráneas) 4.1.1 Fundamentos teóricos de la técnica}

La velocidad horizontal del flujo subterráneo se obtiene a partir de la disminución de la concentración del trazador inyectado en función del tiempo, en un determinado volumen de un pozo, como consecuencia del flujo que circula en dirección perpendicular al mismo. 


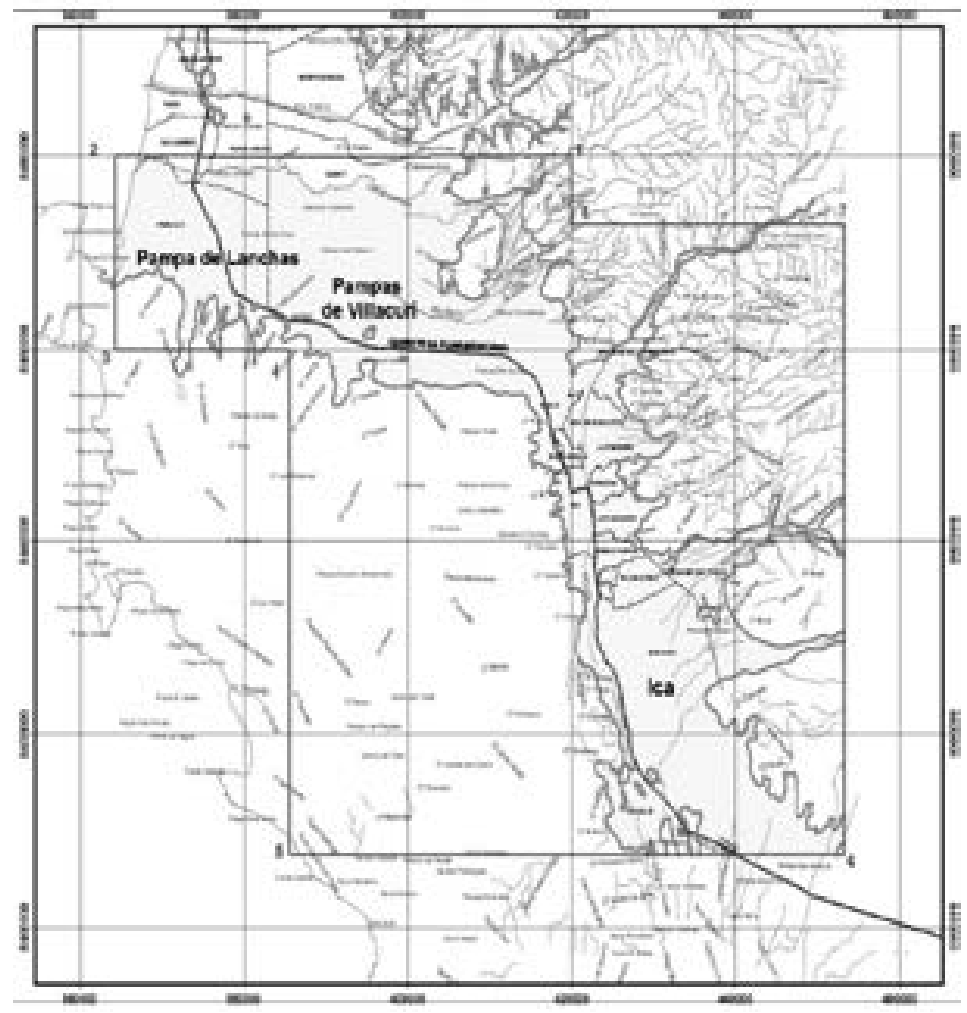

Fig. 1a. Zona de estudios del proyecto integral, que comprende los acuiferos de Ica, Villacuri y Lanchas, ubicados en la costa central del Perú, aproximadamente entre los kilómetros 239 y 340 de la carretera Panamericana Sur. El acuifero Pampa Lanchas (donde se desarrollarán las experiencias), se localiza en la provincia de Pisco $y$ departamento de Ica. Comprende los distritos de Paracas y parte de los distritos de Humay, San Andrés y Túpac Amaru que se encuentran sobre la margen izquierda del rio Pisco.

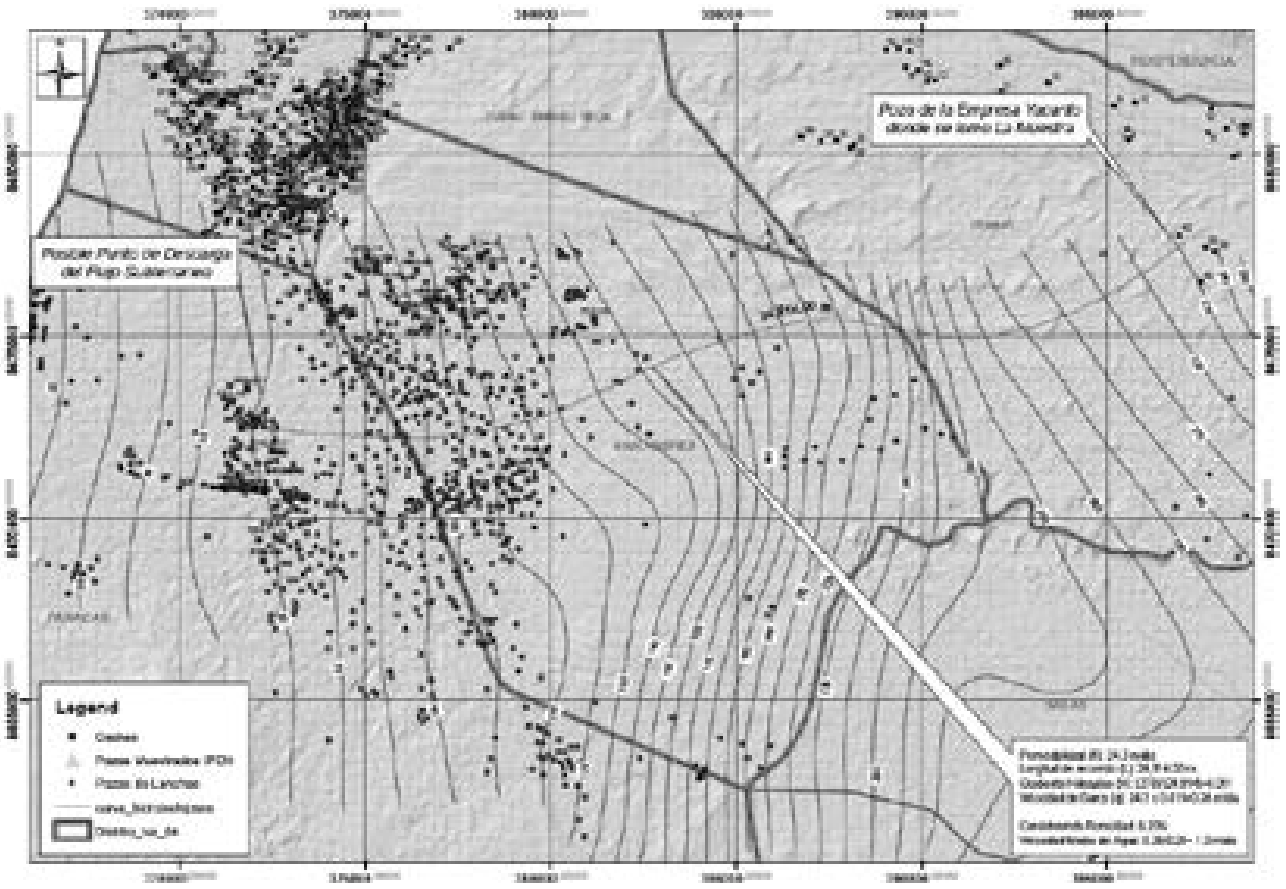

Fig. 1b. Área de estudio especifica en el acuifero Pampa de Lanchas. 
Supongamos que en un tramo de la columna de agua de un pozo de altura h y diámetro d definido por dos "packers» de cierre perfecto, se inyecta cierta cantidad de trazador, lo cual resulta en una concentración inicial Co. Se supone que se cumplen las siguientes condiciones:

a) El flujo de agua que circula a través del pozo tiene un régimen estacionario.

b) En el volumen cilíndrico $\mathrm{V}=\pi \mathrm{d}^{2} / 4$ del tramo considerado, la distribución del trazador se conserva homogénea. Es decir, en cualquier momento, la concentración en todos los puntos de este volumen es la misma. Ello implica que se cumple la condición de buena mezcla o de mezcla instantánea del agua que penetra en el volumen $\mathrm{V}$.

c) La salida del trazador del volumen V sólo tiene lugar como consecuencia del flujo horizontal del acuífero.

En ausencia de flujo vertical y de pérdidas significativas de trazador por difusión osmótica, es decir, cuando existe una difusión derivada del gradiente de concentración existente entre el agua del pozo y el agua del acuífero, la concentración de trazador en el volumen $\mathrm{V}$ disminuye en función del tiempo según la ecuación diferencial siguiente:

$$
\frac{d C}{d t}--\frac{C}{V} \frac{d V}{d t}-Q \frac{C}{V}
$$

El término $\mathrm{dV} / \mathrm{dt}=\mathrm{Q}$ en la ecuación 1 , representa el caudal de agua que circula a través de la sección $S=h \cdot d$. Integrando la ecuación (1), se consigue lo siguiente:

$$
C_{\mathrm{t}}=C_{0} \exp \left(-\frac{Q}{V} t\right) \text { y par tanio: } Q=\frac{V}{t} \ln \frac{C_{e}}{C_{\mathrm{t}}}
$$

Ahí, $\mathrm{C}_{\mathrm{o}}$ es la concentración inicial del trazador en el volumen $\mathrm{V}$ y C, la misma al cabo del tiempo t. Si se tiene en cuenta que el caudal $Q$ es igual al producto $v_{s} \cdot S$, siendo $v_{s}$ la velocidad del flujo que circula dentro del pozo a través de la sección $S$, y que el volumen $V$ equivale a $V=\pi \mathrm{d}^{2} h / 4$, se llega a la ecuación 3:

$$
V_{s}=\frac{\Pi \cdot d}{4 t} \ln \frac{C_{0}}{C_{t}}
$$

Por otra parte, la velocidad dentro del pozo $\mathrm{v}_{\mathrm{s}}$ se relaciona con la velocidad de filtración. Por ello, se necesita introducir un coeficiente que responda a la perturbación hidrodinámica ocasionada por el pozo en el flujo subterráneo.

El valor de este coeficiente puede calcularse o estimarse a partir de los datos constructivos del pozo y de las permeabilidades del acuífero, relleno de grava y tubería de revestimiento. Su valor suele oscilar entre 1 y 4.

Si consideramos, para la relación Co/Ct, un valor igual a 10, se llega a la ecuación 5:

$$
V_{f}=\frac{\Pi \cdot d}{4 x_{1 / 10}} \ln 10=\frac{1,81 d}{\imath a t_{1 / 10}}
$$

En ella, $\mathrm{t}_{1 / 10}$ es el tiempo necesario para que la concentración de trazador en el volumen $\mathrm{V}$ se reduzca al $10 \%$ de su valor inicial, es decir, para que se cumpla $\mathrm{C}_{\mathrm{o}} / \mathrm{C}_{\mathrm{t}}=10$. La ecuación anterior es una fórmula práctica que permite determinar $\mathrm{v}_{\mathrm{f}}$ en función de $\mathrm{t}_{1 / 10}$. Este último tiempo se determina experimentalmente midiendo la variación de la concentración en función del tiempo por medio de un detector apropiado que se sitúa dentro del volumen $\mathrm{V}$ del pozo. 


\subsubsection{Equipo y materiales requeridos para medir la velocidad del flujo de aguas subterráneas}

\section{Para la utilización de un trazador radiactivo:}

1. Un detector-analizador de radiación gamma, con sonda de hasta 100 metros de longitud,

2. Un equipo de inyección de trazadores en pozos,

3. Materiales y herramientas varias para manipular trazadores radioactivos,

4. Trazador radioactivo I-131,

5. Detectores portátiles de radiación gamma.

\section{Para la utilización de un trazador químico (salmuera):}

1. Termoconductivímetro,

2. Sonda de conductividad, con cable adecuado para un máximo de $50 \mathrm{~m}$ de profundidad,

3. Materiales y herramientas básicas para preparación y manipulación del trazador a inyectar,

4. Trazador (sal común).

\subsubsection{Técnica experimental}

La técnica experimental para medición de la velocidad de flujos de agua subterránea se basa en el método de marcado de toda la columna. Este consiste en inyectar, en la columna de agua del pozo, un trazador soluble y fácilmente detectable, y monitorear su desplazamiento en otros pozos cercanos o en el que ha sido inyectado. El método asume que la velocidad del trazador sea igual o bastante similar a la velocidad del de agua.

Para el caso en que se disponga de dos o más pozos, el tiempo transcurrido entre la inyección y la detección del trazador, junto a la información de la distancia existente entre los pozos de inyección y detección, permitirá determinar directamente la velocidad promedio y dirección del flujo de aguas subterráneas. En el caso de que la detección del trazador demore (lo cual podría ocurrir si la velocidad de las aguas subterráneas es muy baja o si las líneas de flujo no enlazan los puntos de inyección y de monitoreo), se puede determinar indirectamente la velocidad y dirección del flujo con la técnica del pozo único.

Esta última consiste en la inyección del trazador en el pozo y en monitorear su concentración durante un periodo que puede ser de horas o días, dependiendo de las características hidrogeológicas del acuífero. Si existe un flujo de agua subterránea que atraviesa el pozo, la concentración del trazador en el pozo disminuirá con el tiempo. La relación de concentraciones inicial y final, el tiempo transcurrido, la geometría del pozo y el tiempo de vida media del trazador (si se usa uno radioactivo) permitirán determinar la velocidad del flujo.

Se debe obtener información cualitativa rápida $y$, a veces, semicuantitativa sobre los flujos existentes a lo largo de la columna de agua del pozo. El método se basa en el marcado de toda la columna de agua del pozo con alguno de los trazadores indicados.

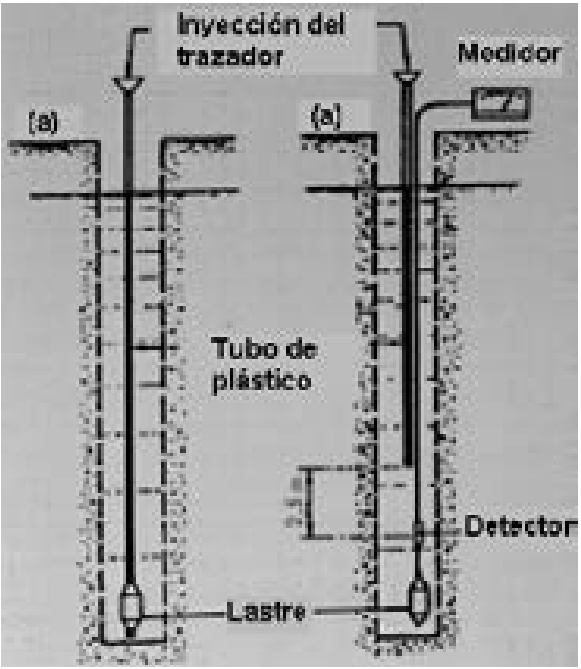

Fig.2. Métodos de marcado de toda la columna de agua en pozos. 
El procedimiento de marcado se ilustra en la figura 2 (en el lado izquierdo). Una manguera de plástico de 8-10 mm de diámetro interior se introduce hasta el fondo del pozo con la ayuda de un pequeño lastre colgado de su extremo inferior.

La manguera está abierta por ambos extremos. Por el superior, se inyecta un volumen de la solución de trazador igual al volumen interno de la misma, medido desde el nivel piezométrico hasta el fondo del pozo. A continuación, se extrae lentamente la manguera a una velocidad aproximadamente constante, con lo cual el trazador queda distribuido con uniformidad en toda la columna de agua.

La inyección puede hacerse por gravedad o por medio de una pequeña bomba. Supongamos que se utiliza como trazador una solución saturada de sal común, cuya solubilidad en agua es de unos $350 \mathrm{~g} / \mathrm{l}$.

La conductividad aproximada de esta solución debe ser del orden de $467 \mathrm{mS} / \mathrm{cm}$. Por otra parte, la sección interna de la manguera de $1 \mathrm{~cm}$ de diámetro es de $0,785 \mathrm{~cm}$. De esta manera, si la inyección se realiza en un pozo de $6 \mathrm{~cm}$ de diámetro, cuya sección es $28,3 \mathrm{~cm}^{2}$, la dilución producida asciende a $28,3 / 0,785=36$. Por tanto, la conductividad del agua del pozo se incrementará en un valor de $467 / 36=13 \mathrm{mS} / \mathrm{cm}$. Este aumento de conductividad es suficiente para realizar el ensayo con comodidad si la conductividad inicial del agua del pozo es inferior a $5 \mathrm{mS} / \mathrm{cm}$.

Si se trata de un pozo de gran diámetro, caso poco frecuente en estudios de fugas de embalses, puede ser necesario utilizar una manguera de diámetro mayor o realizar la inyección del trazador por medio de una bomba peristáltica.

En este caso, se calcula primero el volumen de solución de la sal común que se precisa inyectar en el pozo para que, una vez distribuido por toda la columna, se alcance la conductividad deseada. Este volumen de solución se coloca dentro de un tanque o recipiente apropiado. Luego, se inyecta por medio de la bomba desplazando la manguera verticalmente hacia arriba y hacia abajo hasta que haya sido todo inyectado. La técnica no necesita que la distribución del trazador sea perfecta. Una vez realizada la inyección del trazador, se inician las medidas de sucesivos perfiles de concentración barriendo toda la columna con el detector en saltos de, por ejemplo, 1 o 2 metros, o bien de forma continua si se dispone de un equipo accionado con un motor.

La frecuencia de los perfiles tiene que ser elegida de acuerdo con la velocidad de dilución del trazador. Lo normal es comenzar con una frecuencia muy alta, por ejemplo, de cinco minutos, la cual se modifica posteriormente en función de la velocidad de dilución del trazador.

Ocasionalmente, la velocidad del flujo subterráneo puede ser tan elevada que la mayor parte del trazador escapa del pozo en el intervalo de tiempo que transcurre entre la extracción de la manguera y la introducción de la sonda de medida. Esto ocurre con cierta frecuencia en estudios de fugas de embalses, debido a los elevados gradientes hidráulicos que suelen existir.

En este caso (que se aprecia a la derecha de la figura 2), se introduce, en el pozo, la sonda de medida y la manguera de inyección unidas por cinta adhesiva. El extremo inferior de la manguera se sitúa por encima del detector a una distancia de, por ejemplo, 2,5 metros.

La manguera se llena de solución de trazador de la forma indicada anteriormente. A continuación, se eleva el conjunto manguera y detector en un tramo de $5 \mathrm{~m}$. Cuando se realiza esto, se marca un tramo de columna de agua del pozo igual, asimismo, a $5 \mathrm{~m}$ y el detector queda situado, aproximadamente, en el centro del tramo marcado.

Si mantenemos fijos, en esa posición, la manguera y el detector, se mide el cambio de la concentración del trazador en función del tiempo. Como el flujo es muy rápido, este último escapa después de unos minutos. Una vez finalizada la medida, se eleva el conjunto que conforman manguera y detector otros $5 \mathrm{~m}$ y se repite la medida. El proceso continúa del mismo modo hasta barrer todo el tramo afectado por el flujo rápido. 


\subsubsection{Interpretación de los resultados para medir la velocidad de flujo}

Obviamente, el método solo proporciona la velocidad horizontal del flujo subterráneo cuando no existe flujo vertical al interior del pozo. Por tanto, debido a la elevada frecuencia con que se presentan los flujos verticales, solamente en casos limitados se llega a un valor fiable de velocidad horizontal. Generalmente, esto sucede cuando se trata de pozos de escasa profundidad (columna de agua de pequeño espesor). En aquellos que presentan una gran penetración dentro del acuífero, se obtienen, de manera frecuente, flujos verticales. Asimismo, los de este tipo son habituales en el caso de acuíferos de rocas consolidadas fracturadas o karstificadas cuando los pozos atraviesan dos o más niveles de fracturación o de karstificación. Sin embargo, incluso en estas ocasiones, la técnica proporciona información sobre la localización de los principales horizontes permeables atravesados por el pozo e indica las zonas de entrada y salida del agua.

Se puede evidenciar con facilidad que, para poder interpretar los resultados de forma correcta, es preciso informarse sobre los aspectos constructivos del pozo.

Los parámetros básicos que se necesitan para tal fin son el diámetro interno del mismo, las características del ranurado realizado en la tubería de revestimiento y los datos sobre el posible relleno anular de grava. La figura 3 muestra los tipos de perfiles que suelen obtenerse con esta técnica.

En el caso de la parte a de la figura 3, se puede apreciar un flujo horizontal en el tramo intermedio del pozo. Los sucesivos perfiles de concentración del trazador muestran la disminución del mismo dentro del tramo indicado. En el caso de la parte b, existe un flujo vertical descendente, con la entrada de agua localizada en el tramo indicado con la letra E y la salida en la letra S.

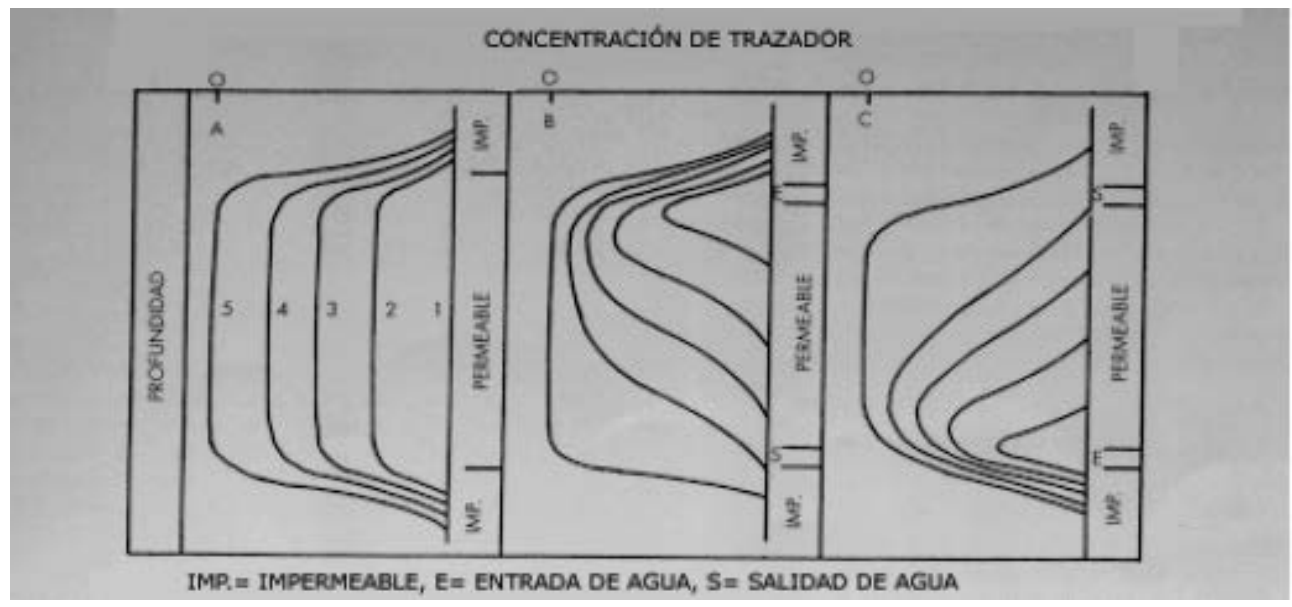

(a)

(b)

(c)

Fig. 3. Tipos de perfiles que se obtienen en el marcado de toda la columna de agua de pozos. a=flujo horizontal; $b=$ flujo vertical descendente; $c=$ flujo vertical ascendente. E=entrada de agua; $S=$ salida del agua.

Los sucesivos perfiles de concentración muestran como esta disminuye de forma progresiva entre ambos tramos permeables.

Las pérdidas de trazador que se producen por encima del E y por debajo del S son consecuencia de la turbulencia creada en el interior del pozo por el flujo de agua. Debe indicarse que, cuando se utiliza sal común como trazador, la salinidad del agua y, por tanto, su densidad, aumentan como consecuencia de la inyección de aquella. 
Por tanto, cuando el agua se renueva en el tramo comprendido entre E y $S$, entra agua menos salina en el pozo y se crea una interfase de aguas con distinta densidad en el techo del tramo E.

Como consecuencia, el agua de mayor densidad existente por encima de este punto tiende a descender por efecto de la densidad y el pozo se «limpia» de trazador en dicho tramo, aunque ello sucede con una velocidad menor que en el tramo afectado por el flujo vertical.

En cambio, en la interfase que se forma en el muro del tramo $S$, la columna de agua es estable, porque el agua de mayor densidad se encuentra a una profundidad mayor. El caso de la parte c es igual que el de la b, solo que el flujo vertical es ascendente. En ambos casos, la velocidad puede determinarse de forma aproximada gracias al descenso o ascenso de los frentes de trazador dentro del pozo, es decir, a partir de las distancias correspondientes a concentraciones iguales de dos frentes sucesivos.

Estas distancias, divididas por el intervalo de tiempo transcurrido entre la medida de los dos perfiles, proporcionan la velocidad del flujo vertical. Obviamente, los perfiles de temperatura y conductividad, medidos antes de inyectar el trazador, son siempre de gran ayuda para interpretar los resultados de los ensayos de marcado de toda la columna de agua.

\subsection{Para las mediciones de la dirección del flujo de aguas subterráneas}

\subsubsection{Fundamentos metodológicos}

La metodología que se aplicará consiste en la inyección, en los pozos investigados, de un trazador que tenga propiedades de adsorción en metales para monitorear su concentración en el perímetro interior del pozo. Esto último requiere de una malla metálica cilíndrica que antes debe ser colocada dentro del pozo y fijada con una orientación determinada. Después de algunas horas, la malla metálica se retira del pozo y se procede a medir la radioactividad en su superficie. Las zonas de la malla que registren mayor concentración radioactiva marcarán las direcciones del flujo de las aguas subterráneas.

\subsubsection{Condiciones de campo requeridas para medir la dirección del flujo de aguas subterráneas}

La zona de estudio debe tener, al menos, un pozo vertical que atraviese el nivel freático de las aguas subterráneas cuya velocidad y dirección de flujo se desea investigar. Los pozos deben tener paredes estables o estar forrados en toda su longitud con una tubería ranurada de un diámetro interior menor al del detector del trazador, que por lo general es del orden de 3 pulgadas.

Los trazadores pueden ser químicos o radioactivos. Estos tienen la ventaja de poder monitorear directamente en el campo y obtener resultados inmediatos. Los primeros, en cambio, requieren de análisis en laboratorio y la obtención de los resultados demora más. Los radioactivos mas usados son el I-131 y el Au-198. Para el presente caso, se utilizó el Au-198, debido a la experticia generada en el IPEN para estas determinaciones y a la confiabilidad de los resultados obtenidos en las aplicaciones previas, en las que se ha utilizado el mencionado radiotrazador producido en el Centro Nuclear de Huarangal, perteneciente a dicha entidad.

\subsubsection{Equipo y materiales requeridos para medir la dirección del flujo de aguas subterráneas}

1. Un detector-analizador de radiación gamma, con sonda de hasta 100 metros de longitud,

2. Un equipo de inyección de trazadores en pozos,

3. Materiales y herramientas varias para manipular trazadores radioactivos,

4. Trazador radioactivo Au-198 e I-131,

5. Detectores portátiles de radiación gamma,

6. Malla metálica. 


\section{POZO - VISTA EN PLANTA}

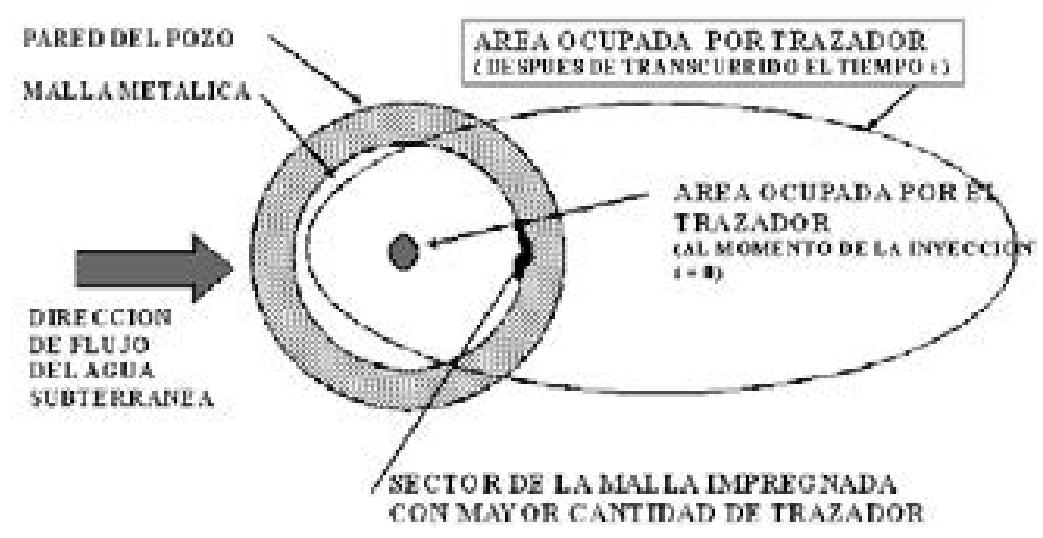

(a)

\section{POZO - VISTA EN CORTE}

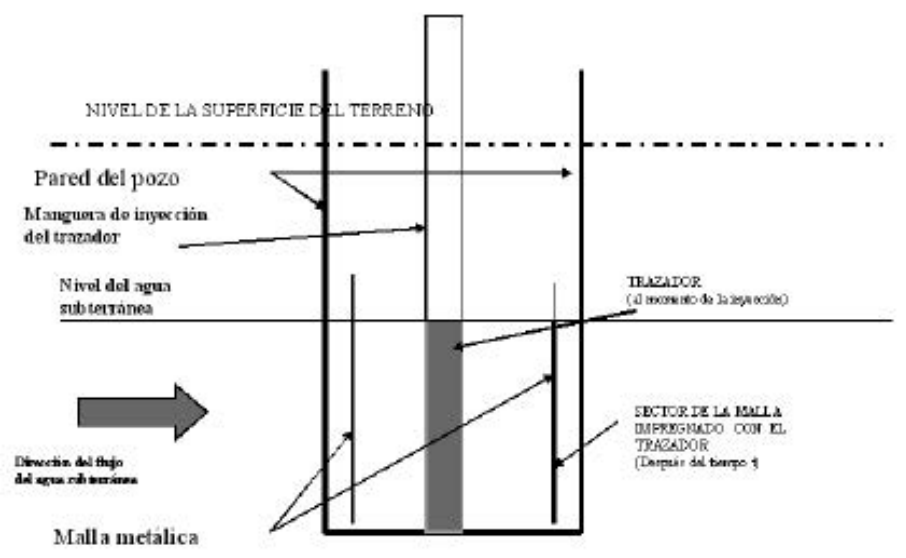

(b)

Fig. 4 a y fig. 4b. Arreglo experimental para la medida de la dirección del flujo de aguas subterráneas 


\section{REFERENCIAS}

[1] Custodio, E. (2010) Hidrología Subterránea (Vol.1). Barcelona: s. e.

[2] Glover, R. (2010) Transient Groundwater Hydraulics. (3 ed.). Colorado: Water Resources Publications.

[3] International Atomic Energy Agency (1997) Emerging New Applications of Radiotracers in Industry. Viena: s. e.

[4] International Atomic Energy Agency (1999) Radiotracer Technology for Engineering Unit Operation Studies and Unit Processes Optimization. Polonia: Krakow-Poland.

[5] Kresic, N. (2007). Hydrogeology and Groundwater Modeling. Florida: CRC Press.

[6] Leclerc, J. P. (2001) Traceurs and tracing methods. Récents Progrès en Génie des procédés, 15(79). Nancy-France: SFGP.

[7] Leclerc, J. P. y Grevillot, G. (1998). Traceurs et méthodes de traçages. Récents Progrès en Génie des procédés, 12(61). Nancy-France: SFGP.

[8] Leclerc, J. P., Mesnier, R., Sebastian, C., Maghella, G., Mamani, E. (2007) Interpretation of radiotracer experiments in an industrial battery of desanders with simultaneous stochastic and non-stochastic flows. Applied Radiation and Isotopes, 65(11), 1208-1214.

[9] Maggio, G. E., Sebastian, C., Duran, O., Cano, M. y Griffit, J. (2000). Guía para aplicaciones industriales de radiotrazadores y fuentes de radiación. Viena: International Atomic Energy Agency.

[10] Nazeer, A. (2014) Hydraulics of Wells Design, Construction and Testing. Virginia: American Society of Civil Engineers

[11] Sebastian, C., Maghella, G. y Mamani, E. (2000) Overview of radiotracer experiments for better understanding of wastewater and water treatment plants in Lima (Peru). Viena: IAEA.

[12] Sebastián C., Maghella G. y Mamani, E. (2004). Evaluación de unidades de tratamiento de agua utilizando técnicas de trazadores. Lima: IPEN, ICT.

[13] Vela, R. (1996). Análisis de la hidrodinámica de sistemas de flujo continuo utilizando trazadores radiactivos. Lima: IPEN. 